\title{
ANÁLISE DA VARIABILIDADE ESPECTRAL NO MONITORAMENTO DA CANA-DE-AÇÚCAR USANDO MEDIDAS DE COMPLEXIDADE E IMAGENS RAPIDEYE
}

\author{
Maristella Cruz de Moraes ${ }^{(a)}$, Luiz Eduardo Vicente ${ }^{(\mathrm{b})}$, Sérgio Henrique V. Leme de Mattos ${ }^{(\mathrm{c})}$ \\ Daniel Gomes dos Santos WendrinerLoebmann ${ }^{(\mathrm{d})}$ \\ (a)IGE/ Instituto de Geociências, Universidade Estadual de Campinas, maristellacruz@gmail.com \\ (b)Embrapa Meio Ambiente/Plataforma ABC, Jaguariúna, SP, Brasil, luiz.vicente@embrapa.br, \\ (c) Faculdade de Ciências Humanas/ Universidade Federal de Grande Dourados, sergiomattos@ ufgd.edu.br \\ (d) Embrapa Meio Ambiente/Plataforma ABC, Jaguariúna, SP, Brasil, daniel.gomes@embrapa.br
}

\section{Eixo: GEOTECNOLOGIAS E MODELAGEM ESPACIAL EM GEOGRAFIA FÍSICA}

\begin{abstract}
Resumo
A constelação Rapideye, operacional desde 2009 com seus cinco satélites, oferece imagens multiespectrais com consideráveis resoluções temporais e espaciais $(5$ dias e $5 \mathrm{~m}$, respectivamente). O sensor REIS foi o pioneiro a oferecer informações sobre a banda Red Edge, situada entre a banda do vermelho e infravermelho próximo, permitindo análises mais robustas no que tange à presença de compostos biofísicos na vegetação, sobretudo de clorofila. Partindo deste pressuposto e da, ainda pouca, utilização deste satélite para a agricultura, analisamos a variabilidade espectral através de métricas de complexidade embasadas em entropia informacional para as bandas 3, 4 e 5 do sensor em fragmentos de diferentes tipos de manejo de cana-de-açúcar na Estação experimental de citricultura de Bebedouro (SP). Os resultados mostram valores coerentes com o arcabouço teórico, considerando incremento informacional (maior complexidade) quando do uso da banda RedEdge, reforçando o uso de imagens Rapideye para tais finalidades, propondo análises alternativas de variabilidade.
\end{abstract}

Palavras chave: Variabilidade, Complexidade, Cana-de-Açúcar, Rapideye, Clorofila.

\section{Introdução}

No que concerne à análise da paisagem e suas dinâmicas, as métricas de paisagem possuem cada vez mais indiscutível aplicação e importância. São resultantes da busca do homem em compreender os processos de formação e capacidade de auto-organização dos sistemas e estão em constantes processos de incremento. Neste sentido, os algoritmos contidos no software Compplexus (VEDOVATO et al., 2013), o qual é utilizado nesse trabalho,foram desenvolvidos e trabalhama partir de métricas embasadas em entropia informacional, a fim de mensurar a complexidade de padrões vegetacionais conforme estudos publicados anteriormente (VEDOVATO et al., 2015; MORAES et al., 2015; ROCHA et al., 2015).

Considerando a agricultura como uma das principais bases econômicas do país e a crescente demanda por tecnologias em seu gerenciamento, faz-se necessário mapeamentos e análises alternativas, especialmente considerando técnicas de sensoriamento remoto, com dados pronto uso, fidedignos, de cada vez mais fácil acesso e baixo custo. As imagens Rapideye são uma alternativa robusta de última 
geração, por terem boa resolução radiométrica (12 bits), temporal ( $\sim 5$ dias) e espectral (5 bandas), trazendo ainda o pioneirismo ao oferecer informações sobre a banda Red Edge (borda do vermelho) permitindo uma melhor discriminação da vegetação por sua alta sensibilidade parâmetros bioquímicos, sobretudo a clorofila. Pensando nisso e na ainda pouca utilização deste sensor para fins agrícolas, o presente estudo pretende testar sua eficiência ao analisar a variabilidade espectral de campos experimentais de cultura de cana-de-açúcar, especialmente nesta faixa do espectro, a fim de ampliar e difundir suas aplicações práticas para além do conhecido.

\section{2. Área de Estudo}

A área de estudo do presente trabalho se encontra nos campos de cultivo da Estação Experimental de Citricultura do município de Bebedouro/SP, área mantida pela Coopercitrus Cooperativa de produtores rurais e conhecida préviamente em outros estudos. A estação dispõe de 5 campos experimentais de cana-de-açúcar, sendo que para cada um deles, um sistema de manejo diferente relacionado à irrigação e adubação é aplicado. Há um sexto campo, que serve como o "não monitorado". São eles: 1 Irrigado; 2 - Não irrigado; 3-MEIOSI; 4- Manejo Convencional; 5- Gestão de Coopercitrus.
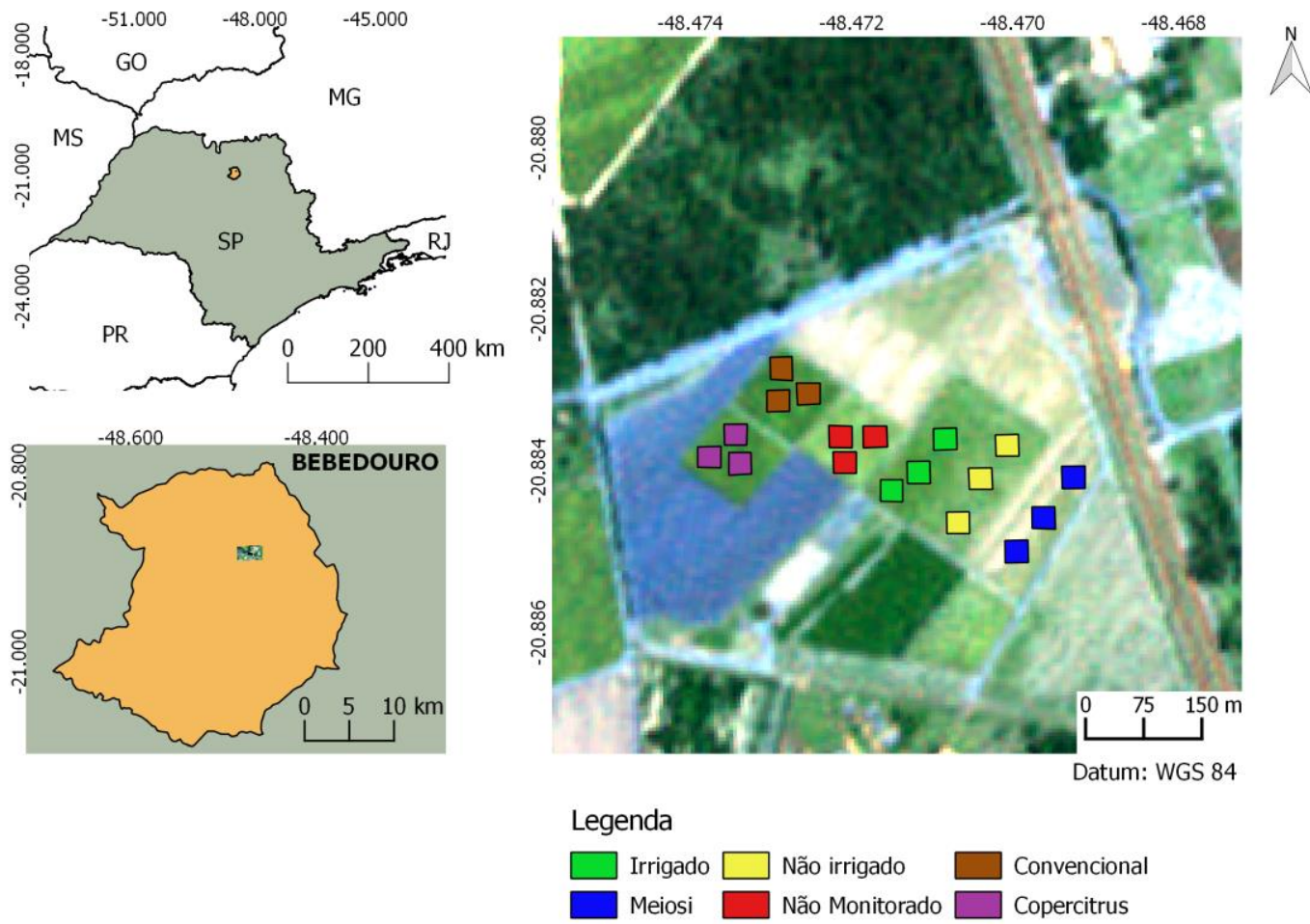

Figura 1 - Área de estudo e amostras extraídas. Imagem Rapideye composta em R:1 G:2 B3.

\section{Materiais e Métodos}


A cena Rapideye em questão é datada de 7 de Abril de 2016, sendo que a colheita seria realizada em 17 de Junho do mesmo ano. Para efeitos qualitativos, a imagem passou por efeitos de correção atmosférica através do código MODTRAN (Moderate Resolution Atmospheric Radiative Transfer Model) considerando parâmetros locais da atmosfera baseado em dados MODIS (Gomes et al., 2013), a fim de converter os dados para reflectância de superfície. Foram selecionados na imagem 3 polígonos regulares de 6x6 pixels (dimensão adequada para bons resultados conforme estudos de MORAES et al $(2014,2015))$ em cada parcela do campo experimental,totalizando 18 polígonos. Destes polígonos foram extraídos os relatórios estatísticos das bandas para cada amostra (ferramenta Roi Tool do software Envi ${ }^{\circledR}$ (ESRI)) exportando-os no formato ASCII para o software Compplexus (VEDOVATO et al., (2013)) a fim de que os cálculos necessários sejam feitos para se obter os valores de complexidade resultantes em cada amostra. A fim de mensurar tais valores de complexidade, o software trabalha com métricas embasadas em entropia informacional. São elas:

- Medida de Variabilidade (He/Hmax): Considera o aumento da complexidade em função do aumento da desordem de um sistema (SHINER et al., (1999). Os fatores a serem considerados no cálculo são a extensão do sistema, a entropia máxima (Hmax) e a entropia informacional (He). Desta forma, a razão entre elas é a medida de complexidade conforme apontam PIQUEIRA \& MATTOS (2011)

$$
C=\frac{H e}{H \max }
$$

- Medida SDL (Shiner, Davison e Landsberg): Aqui a complexidade se encontra num pico de heterogeneidade intermediária. Ou seja, entre o heterogêneo e o homogêneo, como uma função convexa da entropia, usando os mesmos fatores da medida anterior, calculados de forma diferente (PIQUEIRA et al., 2009).

$$
C=\left[1-\left(\frac{H e}{H \max } \cdot \frac{H e}{H \max }\right)\right]
$$

Os relatórios com valores de complexidade gerados são dispostos em valores individualizados para cada banda, e para melhor analisar os resultados, selecionamos as bandas 3, 4 e 5 (Vermelho, Red Edge e Infravermelho próximo respectivamente) que ressaltam/diferenciam melhor as características da vegetação. Destaque para a banda Red Edge, pioneira neste aspecto e situada num intervalo sensível para identificação de diversos níveis de clorofila na vegetação, evitando saturação das imagens (SOUZA et al., 2015 apud. HATFIELD et al., 2008).

\section{Resultados e Discussão}




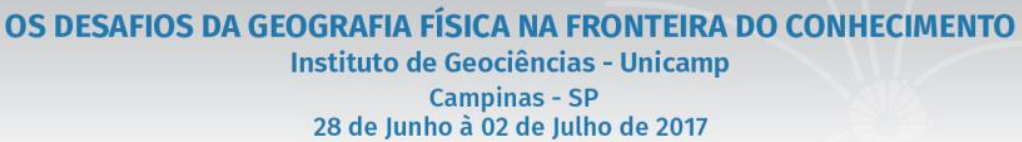

Os resultados foram dispostos em tabela graduada em escala de cores, a fim de facilitar a vizualização

(Tabela 1 - Valores de complexidade graduados das amostras coletadas nas 3 bandas escolhidas)

\begin{tabular}{|c|c|c|c|c|c|c|}
\hline & $\begin{array}{l}\text { Var. } \\
\text { He/Hmax }\end{array}$ & & & SDL & & \\
\hline Amostras/Plantio & RED & Red Edge & NIR & RED & Red Edge & NIR \\
\hline Convencional1 & 0,960916165 & 0,98954056 & 0,994368715 & 0,037556288 & 0,010350044 & 0,005599573 \\
\hline Convencional2 & 0,948132406 & 0,97712663 & 0,997092465 & 0,049177346 & 0,022350179 & 0,002899081 \\
\hline Convencional3 & 0,985346736 & 0,98954056 & 0,994368715 & 0,014438545 & 0,010350044 & 0,005599573 \\
\hline Coopercitrus1 & 0,981400029 & 0,99184525 & 0,997092465 & 0,018254012 & 0,008088252 & 0,002899081 \\
\hline Coopercitrus2 & 0,989905775 & 0,98990577 & 0,984613764 & 0,009992332 & 0,009992332 & 0,0151495 \\
\hline Coopercitrus3 & 0,982968937 & 0,96814079 & 0,994368715 & 0,016741006 & 0,030844201 & 0,005599573 \\
\hline Irrigado1 & 0,98416114 & 0,98567343 & 0,997092465 & 0,015587991 & 0,014121323 & 0,002899081 \\
\hline Irrigado2 & 0,968305611 & 0,98567343 & 0,997092465 & 0,030689855 & 0,014121323 & 0,002899081 \\
\hline Irrigado3 & 0,982968937 & 0,98567343 & 0,991845248 & 0,016741006 & 0,014121323 & 0,008088252 \\
\hline Meiosi1 & 0,987688337 & 0,99436872 & 0,983242855 & 0,012160086 & 0,005599573 & 0,016476343 \\
\hline Meiosi2 & 0,997092465 & 0,98768834 & 0,997092465 & 0,002899081 & 0,012160086 & 0,002899081 \\
\hline Meiosi3 & 0,985346736 & 0,99184525 & 0,991845248 & 0,014438545 & 0,008088252 & 0,008088252 \\
\hline Não monit1 & 0,991845248 & 0,99184525 & 0,994368715 & 0,008088252 & 0,008088252 & 0,005599573 \\
\hline Não monit2 & 0,981836017 & 0,98486195 & 0,996344764 & 0,017834052 & 0,01490889 & 0,003641875 \\
\hline Não monit3 & 0,992969593 & 0,99634476 & 1 & 0,00698098 & 0,003641875 & $1,67 \mathrm{E}-15$ \\
\hline Nãoirrigado1 & 0,997092465 & 0,99436872 & 0,997092465 & 0,002899081 & 0,005599573 & 0,002899081 \\
\hline Nãoirrigado2 & 0,994368715 & 0,98534674 & 0,997092465 & 0,005599573 & 0,014438545 & 0,002899081 \\
\hline Nãoirrigado3 & 0,98416114 & 0,99184525 & 0,989540556 & 0,015587991 & 0,008088252 & 0,010350044 \\
\hline
\end{tabular}

As células vermelhas são valores de complexidade menores, as amarelas seriam valores intermediários, e as verdes, valores altos de complexidade. Para ambas as métricas, em coerência com seu arcabouço teórico pode-se observar o seguinte: Para a métrica SDL e seu pico de heterogeneidade intermediária, tem-se os resultados em amarelo, predominantemente presentes na banda Red Edge nos manejos Convencional, Irrigado e Não-monitorado. Isso demonstra a capacidade da banda Red Edge em traduzir a presença de compostos biofísicos e uma possível influência do sistema de gotejamento em maior biomassa aérea resultando por sua vez em maior presença de clorofila, ou seja, informação e, por conseguinte, maior complexidade. Já para a medida de Variabilidade (He/Hmax) observa-se a complexidade máxima na região do NIR, para a maioria dos experimentos. Isso ocorre porque é justamente na banda do infravermelho próximo que ocorre o registro da energia refletida em decorrência da quantidade de biomassa existente, apto para identificação de diferenças entre culturas (JENSEN, 1996). 


\section{Conclusões}

Com este breve estudo pode-se analisar a eficiência das bandas situadas na porção do infrevermelho próximo e, especialmente a banda Red Edge em demonstrar alta sensibilidade para detectar maior variabilidade/níveis de informação em diferentes tipos de manejo agrícola, o que, por conseguinte, pode ser traduzido também com maior capacidade de detecção de anomalias associadas ao cultivo, por exemplo, estresse hídrico vs. baixa produtividade. Não se deve descartar outros testes e verdades terrestres, e deve-se inclusive pensar sobre repetir os testes em questão em conformidade com dados coletados em campo, a fim de comprovar a eficiência do algoritmo, bem como propor uma maneira alternativa de se comparar o comportamento espectral de uma cultura com seu tipo de gestão agrícola. Entretanto, os métodos utilizados nesse trabalho mostraram-se coerentes, efetivos e de fácil aplicação para medidas de eficiência relacionadas a cobertura espectral do sensor sob investigação, bem como da cultura escolhida.

\section{Agradecimentos}

Agradecemos a equipe Planet Labs e a Santigo e Cintra Consultoria, particularmente ao Maurício Meira e Maurício Schiavollini, pelo apoio logístico. Agradecemos também ao Luiz Gustavo (Guru) e Paulo Sérgio, membros da equipe da Estação Experimental de Citricultura de Bebedouro, pelas informações de campo e manejo de lavouras. Finalmente, obrigado à Equipe da Plataforma de Baixo Carbono/ Embrapa Meio Ambiente.

\section{Referências Bibliográficas}

JENSEN. J. R. Introductory digital imageprocessing: a remotesensing perspective. 2. ed. UpperSaddle River: Prentice-Hall, 1996, 318 p.

MORAES, M. C.; LOEBMANN, D. G. S. W.; PAiM, F. A. P., MATTOS, S. H. V. L., ViCENTE, L. E. Avaliação de imagens multiespectrais Landsat/tm5 em radiância e reflectância de superfície na utilização do software CompPlexus. $8^{\circ}$ Congresso Interinstitucional de Iniciação Científica - CIIC 2014. Anais... Campinas SP. 2014

MORAES, M. C.; LOEBMANN, D. G. S. W.; PAIM, F. A. P.; MATTOS, S. H. V. L.; VICENTE, L. E. Mapas de complexidade de fragmentos de Cerrado a partir da Variabilidade espacial de dados do sensor ThematicMapper 5. In: SIMPÓSIO BRASILEIRO DE SENSORIAMENTO REMOTO, 17., 2015, João Pessoa. Anais... São José dos Campos: INPE, 2015. p. 3927-3934.

PIQUEIRA, J. R. C.; MATTOS, S. H. V. L. Note on LMC complexity measure. Ecological Modelling, v. 222, n. 19. p. 3603-3604, 2011. 
PIQUEIRA, J. R. C.; MATTOS, S. H. V. L.; VASCONCELOS-NETO, J. Measuring complexity in three-trophic level systems. EcologicalModelling, v. 220. p. 266-271, 2009.

ROCHA, T. M. G.; VICENTE, L E.; MATTOS, S. H. V. L.; PAIM, F. A. P.; MORAES, M. C. Identificação de padrões vegetacionais em imagens do sensor Rapideye comparado ao sensor Aster utilizando medidas de complexidade. In: $9^{\circ}$ Congresso Interinstitucional de Iniciação Científica - CIIC 2015. Anais... Campinas - SP. 2015

SHINER, J. S.; DAVISON, M.; LANDSBERG, P. T. Simple measure of complexity. Physical Review E, v. 59, n. 2, p. 1459-1464, 1999.

SOUZA, C. H. W. de., LAMPARELLI, R. A. C.; JUSTINA, D. D. D.; ROCHA, J. V. Estudo da banda Red Edge do satélite Rapideye na descriminação da cobertura vegetal. Anais XVII Simpósio Brasileiro de Sensoriamento Remoto - SBSR, João Pessoa-PB, Brasil, 25 a 29 de abril de 2015, INPE p.3612-3618.

VEDOVATO, L. B.; VICENTE, L. E.; PAIM, F. A. P.; PIQUEIRA, J. R. C.; MATTOS, S. H. V. L.; CompPlexus: programa para avaliação de complexidade de padrões em imagens multiespectrais de sensores remotos. In: SIMPÓSIO BRASILEIRO DE SENSORIAMENTO REMOTO, 16., 2013, Foz do Iguaçú. Anais... São José dos Campos: INPE, 2013. p. 6727-6734.

VEDOVATO, L. B.; VICENTE, L. E.; PAIM, F. A. P.; PIQUEIRA, J. R. C.; MATTOS, S. H. V. L. Análise multiespectral de medidas de complexidade utilizando o software CompPlexus aplicado a dados do sensor ASTER. In: SIMPÓSIO BRASILEIRO DE SENSORIAMENTO REMOTO, 17., 2015, João Pessoa. Anais... São José dos Campos: INPE, 2015. p. 5967-5974. 